\title{
Review
}

\section{Prostate cancer: diagnosis and staging}

\author{
Nigel Borley, Mark R. Feneley \\ Department of Urology, University College Hospitals, London NW1 2BU, UK
}

\begin{abstract}
Prostate cancer represents an increasing health burden. The past 20 years, with the introduction of prostate-specific antigen (PSA), has seen prostate cancer move increasingly from a condition that presented with locally advanced disease or metastases to one that is found upon screening. More is also known about the pathology of pre-malignant lesions. Diagnosis relies on trans-rectal ultrasound (TRUS) to obtain biopsies from throughout the prostate, but TRUS is not useful for staging. Imaging for staging, such as magnetic resonance imaging or computed tomography, still has a low accuracy compared with pathological specimens. Current techniques are also inaccurate in identifying lymph node and bony metastases. Nomograms have been developed from the PSA, Gleason score and clinical grading to help quantify the risk of extra-capsular extension in radical prostatectomy specimens. Improved clinical staging modalities are required for more reliable prediction of pathological stage and for monitoring of response to treatments.
\end{abstract}

Asian Journal of Andrology (2009) 11: 74-80. doi: 10.1038/aja.2008.19; published online 1 December 2008.

Keywords: diagnosis, imaging, prostate cancer, PSA, staging

\section{Introduction}

The most remarkable and challenging aspect of prostate cancer diagnosis and staging in the past 20 years or so has been the change from a disease that presented late with locally advanced and metastatic disease to one that is found upon screening or incidentally.

The rise in the number of cases of early-stage disease has brought with it questions regarding the best way to treat these cases.

The biggest drivers of change have been the use of prostate-specific antigen (PSA) as a screening tool and trans-rectal ultrasound (TRUS)-guided biopsy for diagnosis. Now, most cases of prostate cancer are detected by abnormal serum PSA, leading to trans-rectal biopsy. Some cases may still be identified unexpectedly after

Correspondence to: Dr Nigel Borley, University College Hospitals, 235 Euston Road, London NW1 2BU, UK.

Fax: +44-20-7380-9303 E-mail: nigel.borley@uclh.nhs.uk

Received: 29 September 2008 Accepted: 2 October 2008

Published online: 1 December 2008 trans-urethral resection or retropubic prostatectomy for lower urinary tract symptoms. Unfortunately, many cases present with symptomatic locally advanced disease or distant metastases, particularly in men who have not had regular PSA testing. PSA testing may be used as a screening tool in asymptomatic men, as part of the investigation of lower urinary tract symptoms or for the investigation of an abnormal prostatic nodule on digital rectal examination (DRE).

The fact that PSA has become the first widely used screening test for prostate cancer has altered the observed incidence and distribution of the disease. In the early 1990s, countries that used PSA for diagnosis, particularly the United States, noted a steady rise in the incidence of prostate cancer. This has led to concerns over the leadtime bias of detecting cancer earlier in its asymptomatic course and the consequent increase in apparent survival after treatment even without an effect on the natural history of the disease. In addition, there is length-time bias, whereby screening detects preferentially slow-growing tumours, including those of little or no clinical significance .

Although PSA has become, by default, the test for 
detecting early-stage prostate cancer, there remain many questions as to its use as a screening tool and the wider benefit or harm that a prostate cancer screening programme might bring. The Wilson and Junger criteria are used by the World Health Organization (WHO) to judge the merits and effectiveness of the proposed screening programmes and to judge whether general screening of a population should be put in place. These criteria assess the disease, the screening tests, and the impact of the disease's treatments.

According to these criteria, prostate cancer represents an important disease target because it has a large and substantial impact on men's health. However, despite much investigation, the natural history of the disease cannot be reliably predicted in the individual patient and, particularly in elderly men, it may have an innocuous and asymptomatic course.

The PSA test itself is simple, safe and widely acceptable, but it is not efficient because of its high false-positive rate. With regard to the available treatments for early-stage prostate cancer, there is no agreed consensus on the optimal modality and its true efficacy. Current therapies have significant potential side effects and that alone may make any particular treatment unacceptable under individual circumstances. The additional concerns introduced by detecting disease through screening have been mentioned earlier. Several randomized controlled trials of prostate cancer screening are currently underway, and their findings are eagerly awaited, particularly with respect to the impact of screening on mortality and morbidity outcomes.

\section{Diagnosis of prostate cancer}

\subsection{Digital rectal examination}

DRE remains the primary test for the initial clinical assessment of the prostate. DRE was the first screening test to be evaluated and is still routinely used along with PSA testing. It has the benefit of detecting non-PSAsecreting tumours. In the many studies performed since the first investigation of its accuracy in 1956 [1], the positive predictive value of DRE has been around 50\% [2]. However, the DRE is a test with only fair reproducibility, even in the hands of experienced examiners. It 'misses' a substantial number of cancers and, compared with PSA, it detects cancers at a more advanced pathological stage [3]. Indeed, in the pre-PSA era, $75 \%$ of men diagnosed with prostate cancer by DRE ultimately died of their disease [4].

\subsection{PSA}

PSA is a serine protease that was first described in 1979 [5]. It is produced by the prostatic epithelium and periurethral glands and is present in large amounts in prostatic secretions. As a biomarker, it is organ specific, but not cancer specific, and more recently it has acquired some utility as a marker of benign epithelial masses [6]. The first normal reference range for serum PSA was set rather arbitrarily as less than $4 \mathrm{ng} \mathrm{mL}^{-1}$, with a "diagnostic grey area" between 4 and $10 \mathrm{ng} \mathrm{mL}^{-1}$. As PSA production from benign epithelium increases with age, age-specific cutoff points were developed to improve the test's specificity [7]. Age-specific cutoffs reduce the number of biopsies that are performed by $44 \%$, but also result in a $47 \%$ increase in organ-confined disease being missed [8]. While agespecific levels may increase the sensitivity of PSA for detecting cancer in younger men, they reduce test sensitivity, particularly for early-stage and curable disease, in older men.

For improved test specificity, the utility of PSA den-

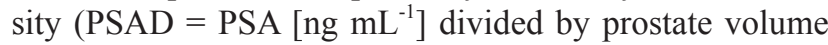
$[\mathrm{mL}])$ has been evaluated. A PSAD of $>0.15$ increased the specificity of detection of prostate cancer compared with total serum PSA, although the optimal cutoff is debated [9]. One study showed that a cutoff of $0.15 \mathrm{ng} \mathrm{mL} \mathrm{m}^{-1}$ would miss $47 \%$ of cancers [10]. Other difficulties include the need to calculate prostate volume, the consistency of measurements and the accuracy of individual volume determinations.

The rate of change of PSA over time (measured as PSA velocity) has been proposed as a means of improving the positive predictive value (PPV) of PSA. An early study reported that an absolute increase of $0.75 \mathrm{ng} \mathrm{mL}^{-1}$ per year or greater increased the test specificity to over $90 \%$ [11]. However, assessment of an increasing PSA requires at least three measurements and an interval between tests of 1.7-2.0 years. The concern is that this may allow for disease progression while the tests are being carried out.

In the blood stream, $70 \%-90 \%$ of circulating PSA is complexed, mostly to $\alpha 1$-antichymotrypsin or $\alpha 2$ macroglobulin; the remaining PSA is referred to as free PSA (fPSA) [12]. Using a cutoff of $25 \%$ for the free/total ratio, a test sensitivity of $95 \%$ may be achieved with $20 \%$ fewer biopsies (i.e., for only $5 \%$ of cancers missed) [13]. The free/total PSA ratio may be particularly useful after previous negative biopsies on a prostate $<40 \mathrm{~mL}$ when a further biopsy is being considered.

fPSA is now known to circulate in different forms, one of which is benign prostatic hyperplasia $(\mathrm{BPH})$ associated PSA (BPSA). BPSA is associated particularly with $\mathrm{BPH}$ and the transition zone [14]. A precursor form of PSA, proPSA, is more associated with cancer. ProPSA, when measured in the range of $2.0-4.0 \mathrm{ng} \mathrm{mL}^{-1}$, has significantly greater cancer detection than either fPSA or total PSA. Test specificity, which is $23 \%$ for total PSA alone, is increased to $44 \%$ when the combination of total PSA, fPSA and proPSA is used [15].

The urinary marker uPM3 detects non-coding mRNA derived from the $P C A 3$ gene that is expressed only in 
prostate tissue and is 60- to 100-fold overexpressed in cancer. It is excreted in the urine and measured in a sample collected after a thorough DRE. uPM3 offers superior specificity and accuracy compared with total PSA and fPSA and has been evaluated mainly for predicting cancer on re-biopsy rather than on primary diagnosis. The result indicates the probability of cancer for the individual patient and is usually interpreted against a cutoff of 35 , but, importantly, it should be used along with other diagnostic tests for prostate cancer.

\subsection{Transrectal ultrasonography and biopsies}

The most common diagnostic modality for prostate cancer is currently TRUS. TRUS provides imaging of the prostate and seminal vesicles using a 7.5-mHz biplane intra-rectal probe measuring $2.5 \mathrm{~cm}$ in diameter. TRUS was historically used as a diagnostic test to identify hypoechoic lesions that may signify cancer. However, $<20 \%$ of hypo-echoic lesions proved to be cancer upon biopsy [16] and $50 \%$ of non-palpable cancers were missed [17]. TRUS is therefore not recommended for the detection of early-stage prostate cancer. It can, however, image the outline of the prostate, identify cysts, abscesses and calcifications within the prostate, and be used to determine prostate volume.

TRUS has established its place in the detection of cancer by its ability to accurately guide needle biopsies and map them to specific regions of the prostate. Patients may find the procedure uncomfortable or occasionally painful, but traditional discomfort has been reduced reliably by the now routine use of local anaesthetic, particularly with the larger number of cores now taken. Local anaesthetic solution is injected adjacent to the posterolateral neurovascular bundles using ultrasound guidance. The procedure is performed on an outpatient basis, with the patient in the lateral decubitus or dorsal lithotomy position. It takes 5-10 min, allowing for 10-12 needle core biopsies to be taken.

The indications for TRUS-guided biopsies include abnormal DRE, an elevated PSA or increasing PSA, and previous biopsies showing isolated prostatic intra-epithelial neoplasia (PIN) or atypical small acinar proliferation (ASAP). Exceptions include elderly or frail men with massively elevated PSA and abnormal DRE, or those in whom a TURP is indicated for BOO with severe LUTS/retention where tissue will be obtained for histological examination. Other indications include the assessment of viable prostate cancer after primary treatment if further treatment is being considered.

The usual biopsy protocol takes $10-12$ needle core biopsies (18 Fr gauge) for systematic mapping of the prostate, including any palpable or radiological target lesions. The historical sextant protocol (a parasagittal base, midgland and apex from each side) has been superseded by the technique of performing 8,10 or 12 biopsies, adding samples from the far lateral peripheral zones. Studies have shown that these extra biopsies detect up to $15 \%$ more cancers. Additional biopsies of the transition zone may be taken if a transition zone cancer is suspected or if a patient is undergoing repeat biopsies because of an increasing PSA. Seminal vesicle biopsies are performed occasionally, particularly for cancer staging when the vesicles appear abnormal on DRE, TRUS or magnetic resonance imaging (MRI), but such staging can be unreliable and therefore has limited clinical utility.

Complications of prostatic biopsy include urinary tract infection, requirement for appropriate antibiotics and occasional septicaemia $(<1 \%)$ necessitating hospital admission. Bleeding (rectal, urinary and/or haemospermia) is usually self-limiting, but very occasionally may be severe and require hospital admission and/or transfusion. Haemospermia may persist for up to 6 weeks. Discomfort or pain may warrant analgesia, but, if severe or persistent, may indicate a significant complication. Biopsy may precipitate voiding difficulty or urinary retention.

It should be noted that it is not safe to biopsy patients when they are on warfarin or have coagulopathies. Aspirin is not generally considered a contraindication, but can be judiciously stopped in anticipation of the procedure, whereas other anti-platelet medications (e.g., clopidogrel) should be stopped 10 days before biopsy.

It is important for the patient to appreciate that negative biopsies do not exclude the possibility of prostate cancer and that a positive result will not necessarily result in the recommendation of immediate treatment.

\subsection{Repeat and saturation biopsies}

Repeat biopsies are indicated after high-grade PIN or ASAP is found, when previous biopsies are normal but the PSA continues to rise, when DRE is suspicious for cancer, or to confirm the presence of viable cancer after treatment if further treatment would be considered.

In one series in which cancer was detected in $57 \%$ of men having prostate biopsy but clinical suspicion of cancer remained after biopsy, in those having repeat biopsies the cancer detection rate fell to $23 \%$ at the second biopsy and $21 \%$ at the third [18]. Although the number of cancer cases detected by repeat biopsy was significant, these tumours were of smaller volume and lower grade.

When clinical suspicion for cancer remains high after initial biopsy, 'saturation' biopsies may sometimes be taken, requiring 20 or more cores. These may be taken throughout the gland or targeted to suspicious areas of the gland that have been seen upon imaging. Compared with the standard biopsy technique, the saturation biopsy technique increases the rate of diagnosis in repeat procedures in patients with previously negative biopsies, but it does not give a significantly greater rate of diagnosis when car- 
ried out as an initial test [19].

\subsection{Ultrasound-guided trans-perineal and template- guided biopsies}

This technique must be performed with the patient in the dorsal lithotomy position. It is reserved for patients who do not have a rectum or require saturation biopsies. It is also used in template-guided biopsies to accurately map the distribution if targeted therapy is proposed for a locaized lesion [20].

Template biopsy requires more extensive analgesia and may be done under general anaesthesia. The areas of the prostate are systemically sampled using a template placed against the perineal skin with a $5-\mathrm{mm}$ grid pattern attached to the TRUS probe. The anterior portion of the gland may be sampled, and separate base and apex samples may be taken. This approach may potentially produce a higher detection rate and more accurate grading [21]. Further advantages of trans-perineal biopsy are that the skin can be better sanitized compared with the rectal mucosa and that the rate of urinary retention is lower [22].

\section{$3 \quad$ Pathology and premalignant lesions}

\subsection{Grading of prostate cancer adenocarcinoma}

The standard grading of prostate cancer is performed according to the Gleason grading system, which was first described by Gleason and Mellinger in 1974 [23]. Although recent modifications have been recommended for consistency and reproducibility among pathologists, this system remains a valuable predictor of cancer behaviour.

The pattern made by the glands is graded on a score from 1 (least aggressive) to 5 (most aggressive) on the largest available histological specimen, either a biopsy or, after radical prostatectomy, a whole prostate. The two most common Gleason patterns are added to give a total score ranging from $2(1+1)$ to $10(5+5)$. The primary and secondary grades are given (e.g., $3+4)$ or, where there is no secondary grade, the primary grade is designated as both the primary and the secondary grade (e.g., 3 +3 ). Occasionally, a tertiary score may be given, which is of higher grade than the primary and secondary grades.

\subsection{Pre-malignant lesions and implications}

Two histological lesions are currently regarded as either pre-malignant or peri-malignant: PIN and ASAP.

\subsection{Prostatic intra-epithelial neoplasia}

PIN consists of architecturally benign prostatic acini and ducts lined by cytologically atypical cells and used to be reported as low-grade (mild) or high-grade (moderate to severe) forms. Pathologists now report only high-grade PIN, because low-grade PIN has no prognostic value.
High-grade PIN is thought to be a precursor for intermediate or high-grade prostate cancer and its finding on biopsies indicates a $30 \%-40 \%$ possibility of prostate cancer at subsequent biopsy.

Currently, it is recommended that repeat or interval systematic biopsies be performed if isolated high-grade PIN is reported on needle biopsy or TURP, bearing in mind the extent of sampling and other clinical factors that may indicate missed cancer [24].

\subsection{Atypical small acinar proliferation}

In ASAP, acini are small and lined with cytologically abnormal epithelial cells with prominent nuclei containing nucleoli. The basal layer may be focally absent, but the basement membrane is intact. Similar to PIN, studies have shown that ASAP at needle biopsy predicts cancer at subsequent biopsy in $>40 \%$ of cases [25]. Currently, it is recommended that repeat systematic biopsies be performed if isolated ASAP is reported on needle biopsy or TURP.

\subsection{Staging of prostate cancer}

The 2002 TNM Classification for Adenocarcinoma of the Prostate is based on T (primary tumour), N (lymph nodes) and $\mathrm{M}$ (metastases) categories. The $\mathrm{T}$ category is based on clinical examination, imaging, endoscopy, biopsy and biochemical tests. The $\mathrm{N}$ category is based on clinical examination or imaging. The $\mathrm{M}$ category is based on clinical examination, imaging, skeletal studies and biochemical tests [26].

The staging is as follows:

\section{Primary tumour}

TX Primary tumour cannot be assessed;

TO No evidence of primary tumour;

T1 Clinically inapparent tumour, neither palpable nor visible by imaging;

T1a Tumour (non-palpable) as incidental histological finding at transurethral resection of prostate in 5\% tissue resected;

T1b Tumour (non-palpable) as incidental histological finding at transurethral resection of prostate in $>5 \%$ of tissue resected;

T1c Tumour (non-palpable) identified by needle biopsy (for elevated serum PSA): includes bilateral nonpalpable tumour on needle biopsy;

T2 Tumour confined within prostate (including prostatic apex, prostate capsule) that is either palpable or visible on imaging or (with p-prefix) demonstrated in radical prostatectomy specimen;

T2a Tumour involving one-half of one lobe or less;

$\mathrm{T} 2 \mathrm{~b}$ Tumour involving more than one-half of one lobe but not both lobes;

T2c Tumour involving both lobes; 
T3 Tumour extends through prostatic capsule;

T3a Extra-capsular extension (ECE);

T3b Invasion of seminal vesicle(s);

T4 Tumour fixed or invades adjacent structures: bladder neck, external sphincter, rectum, levator muscles and pelvic wall;

\section{Regional lymph nodes}

Pelvic lymph nodes are defined as those below bifurcation of common iliac arteries.

NX Regional lymph nodes cannot be assessed;

N0 No regional lymph node metastases;

N1 Regional lymph node metastases within true pelvis, below common iliac artery bifurcation, either unilateral or bilateral;

\section{Metastases}

MX Distant metastases cannot be assessed;

M0 No distant metastases;

M1a Non-regional lymph node metastasis;

M1b Metastasis to bone(s);

M1c Other site(s) of metastasis.

\section{Staging}

Clinical staging is highly important in assessing the risk of the disease for an individual patient and therefore also for treatment recommendations. Clinical $\mathrm{T}$ staging may be done by DRE, but as mentioned previously, it is subjective, with poor reproducibility.

For clinically localized prostate cancer, pathological stage remains the single most important prognostic indicator. Nomograms for predicting pathological $\mathrm{T}$ staging based on pre-operative clinical staging, PSA and Gleason score on biopsy have been well validated [27]. Radiological imaging has not been reliable for accurate pathological staging of the primary tumour and therefore should not be used alone in making therapeutic recommendations.

Recent advances in MRI protocols guarantee improved accuracy both for diagnosis and for staging. MRI may have a role in identifying abnormal regions for core biopsy, either in initial biopsy protocols or on repeat biopsy for patients with previously negative biopsies [28]. Studies using endo-rectal coils have not shown a significant advantage [29]. For staging, features that may help to identify ECE include obliteration of the rectoprostatic angle and asymmetry of neurovascular bundles [29]. Preoperative MRI reports compared with histological specimens at radical prostatectomy have shown a sensitivity of $42 \%$, a specificity of $95 \%$, a positive predictive value of $75 \%$ and a negative predictive value of $84 \%$ [30]. The value of the radiological prediction of extraprostatic invasion relates to the potential therapeutic advantage that has to be gained through achieving negative surgical margins at radical prostatectomy or through choice of alternative therapeutic modalities, both highly controversial.

MRI may have some benefit in predicting seminal vesicle invasion (stage $\mathrm{pT} 3 \mathrm{~b}$ disease), with a positive predictive value of $78 \%$ and a negative predictive value of $94 \%$, and in predicting regional lymph node metastases [31].

\subsection{Lymph node staging}

Regional lymph node metastases are strong predictors of progression [32]. Imaging for lymph node metastases is necessary for men who are at higher risk of metastases, particularly those with palpable disease on DRE, marked elevation of PSA $\left(>20 \mathrm{ng} \mathrm{mL}^{-1}\right)$ or poorly differentiated disease (Gleason grade 4 or 5). The risk of lymph node metastases can be estimated using nomograms.

Computed tomography (CT) and MRI are both poorly able to identify involved nodes because $75 \%$ of metastatic deposits are smaller than the 8- to $10-\mathrm{mm}$ size that can be detected. A meta-analysis has shown that CT and MRI have a sensitivity of $42 \%$ and $39 \%$, respectively, and both have a specificity of $82 \%$ [33]. Techniques using intravenous injection of iron oxide nanoparticles may help improve detection of nodal metastases by characterizing lymph node architecture. This technique increased the sensitivity to $91 \%$ [34].

The historical practice of fine-needle aspiration is not sufficiently accurate for clinical decision making. The gold standard is pelvic lymph node dissection, carried out at the time of radical prostatectomy or as a primary staging procedure before definitive treatment recommendation. An extended lymph node dissection is necessary to detect isolated metastatic lymph nodes that may be present beyond the classical obturator packet [35]. By undertaking an extended node dissection, men at 'high risk' (outlined above) have a $31 \%$ chance of regional metastases.

\subsection{Staging for bone metastases}

Bone scintigraphy has long been used as the mainstay modality for detecting distant metastases in the axial skeleton [36]. At least $25 \%$ of bone metastatic deposits are symptomatic. However, false positives may occur, in association either with recent trauma or with various other non-cancerous sources that may not be resolved by plain radiograph comparison. Fifty percent of bone density must be replaced by tumour before the metastasis can be detected by standard imaging methods [37].

Single photon emission computed tomography (SPECT) has a higher accuracy than bone scintigraphy for vertebral lesions [38] but is not widely used.

MRI is superior to scintigraphy and SPECT in detecting bone metastases in the spine and resolving equivocal scans of the spine, with 39\% more deposits identified [39]; however, non-vertebral skeletal lesions are not usually im- 
aged on MRI.

\section{$5 \quad$ Future directions}

Although new clinical tests for the diagnosis of prostate cancer continue to be developed, current diagnostic priorities must focus on the discrimination between those early-stage tumours that represent a significant threat to life expectancy and quality of life and those that do not. Improved clinical staging modalities are required for more reliable prediction of pathological stage and, more importantly, discrimination of response to alternative therapeutic interventions. Prostate cancer remains a significant health burden, and medical advances must focus on where will be most effective at reducing the high level of mortality and morbidity still associated with this disease.

\section{References}

1 Jewett HJ. Significance of the palpable prostatic nodule. J Am Med Assoc. 1956; 160: 838-9.

2 Stone NN, DeAntoni EP, Crawford ED. Screening for prostate cancer by digital rectal examination and prostate-specific antigen: results of prostate cancer awareness week, 1989-1992. Urology 1994; 44: 18-25.

3 Smith DS, Catalona WJ. Interexaminer variability of digital rectal examination in detecting prostate cancer. Urology 1995 45: 70-4.

4 Gann PH, Hennekens CH, Stampfer MJ. A prospective evaluation of plasma prostate-specific antigen for detection of prostate cancer. JAMA 1995; 273: 289-94.

5 Wang MC, Valenzuela LA, Murphy GM, Chu TM. Purification of a human prostate specific antigen. Invest Urol 1979; 17: 159-63.

6 Andriole G, Djavan B, Fleshner N, Schroder F. The case of prostate cancer screening with prostate-specific antigen. Eur Urol 2004; 5: 737-45.

7 Oesterling JE, Jacobsen SJ, Chute CG, Guess HA, Girman CJ, et al. Serum prostate-specific antigen in a community-based population of healthy men. Establishment of age specific reference ranges. JAMA 1993; 270: 860-4

8 Hakama M, Stenman UH, Aromaa A, Leinonen J, Hakulinen T, et al. Validity of the prostate specific antigen test for prostate cancer screening: followup study with a bank of 21000 sera in Finland. J Urol 2001; 166: 2189-91.

9 Ohori M, Dunn JK, Scardino PT. Is prostate-specific antigen density more useful than prostate-specific antigen levels in the diagnosis of prostate cancer? Urology 1995; 46: 666-71.

10 Catalona WJ, Richie JP, deKernion JB, Ahmann FR, Ratliff TL, et al. Comparison of prostate specific antigen density in the early detection of prostate cancer: receiver operating characteristic curves. J Urol 1994; 152: 2031-6.

11 Carter HB, Morrell CH, Pearson JD, Brant LJ, Plato CC, et al. Estimation of prostate specific growth using serial prostatespecific antigen measurements in men with and without prostate disease. Cancer Res 1992; 52: 3323-8.

12 Lilja H, Christensson A, Dahlen U. Prostate specific antigen in serum occurs predominately in complex with alpha 1-antichymotrypsin. Clin Chem 1991; 37: 1618-25.

13 Catalona WJ, Partin AW, Slawin KM, Brawer MK, Flanigan $\mathrm{RC}$, et al. Use of the percentage free prostate-specific antigen to enhance differentiation of prostate cancer from benign prostatic disease: a prospective multicenter trial. JAMA 1998; 279: 1542-7.

14 Mikolajczyk SD, Millar LS, Wang TJ, Rittenhouse HG, Wolfert $\mathrm{RL}$, et al. "BPSA" a specific molecular form of free prostatespecific antigen, is found predominately in the transition zone of patients with nodular benign prostatic hyperplasia. Urology 2000; 55: 41-5.

15 Khan MA, Partin AW, Rittenhouse HG, Mikolajczyk SD, Sokoll $\mathrm{LJ}$, et al. Evaluation of prostate specific antigen for the early detection of prostate cancer in men with a total specific antigen range of 4.0 to $10.0 \mathrm{ng} / \mathrm{mL}$. J Urol 2003; 170: 723-6.

16 Flanigan RC, Catalona WJ, Richie JP, Ahmann FR, Hudson MA, et al. Accuracy of digital rectal examination and ultrasonography in localising prostate cancer. J Urol 1994; 152; 1506-9.

17 Carter HB, Hamper UM, Sheth S, Sanders RC, Epstein JI, et al. Evaluation of transrectal ultrasound in the diagnosis of prostate cancer. J Urol 1989; 142: 1008-10.

18 Tan N, Lane BR, Li J, Moussa AS, Soriano M, et al. Prostate cancers diagnosed at repeat biopsy are smaller and less likely to be high grade. J Urol 2008; 180: 1325-9; discussion 1329.

19 Lane BR, Zippe CD, Abouassaly R, Schoenfield L, MagiGalluzzi C, et al. Saturation technique does not decrease cancer detection during followup after initial prostate biopsy. J Urol 2008; 179: 1746-50; discussion 1750.

20 Schulte RT, Wood DP, Daignault S, Shah RB, Wei JT. Utility of extended pattern prostate biopsies for tumor localization: pathologic correlations after radical prostatectomy. Cancer 2008; 113: $1559-65$.

21 Megwalu II, Ferguson GG, Wei JT, Mouraviev V, Polascik TJ, et al. Evaluation of a novel precision template-guided biopsy system for detecting prostate cancer. BJU Int 2008; 102: 54650 .

22 Merrick GS, Taubenslag W, Andreini H, Brammer S, Butler WM, et al. The morbidity of transperineal template-guided prostate mapping biopsy. BJU Int 2008; 101: 1524-9.

23 Gleason DF, Mellinger GT. Prediction of prognosis for prostatic adenocarcinoma by combined histological grading and clinical staging. J Urol 1974; 111: 58-64.

24 Lefkowitz GK, Taneja SS, Brown J, Melamed J, Lepor H. Follow-up interval prostate biopsy 3 years after diagnosis of high grade prostatic intraepithelial neoplasia is associated with high likelihood of prostate cancer, independent of change in prostate specific antigen levels. J Urol 2002; 168: 1415-8.

25 Chan TY, Epstein JI. Follow-up of atypical prostate needle biopsies suspicious for cancer. Urology 1999; 53: 351-5.

26 TNM Classification of Malignant Tumours, 6th edn. New York: Wiley-Liss; 2002.

27 Partin AW, Mangold LA, Lamm DM, Walsh PC, Epstein JI, et al. Contemporary update of prostate cancer staging nomograms (Partin tables) for the new millennium. Urology 2001; 58: 843-8.

28 Yeun JS, Thng Ch, Tan PH, Khin LW, Phee SJ, et al. Endorectal magnetic resonance imaging and spectroscopy for the detection of tumour foci in men with prior negative transrectal ultrasound prostate. J Urol 2004; 171: 1482-6. 
29 Yu KK, Hricak H, Alagappan R, Chernoff DM, Bacchetti P, et al. Detection of extracapsular extension of prostate carcinoma with endorectal and phase-array coil MR imaging: multivariate feature analysis. Radiology 1997; 2002: 697-702.

30 Wang L, Mullerad M, Chen HN, Eberhardt SC, Kattan MW, et al. Prostate cancer: incremental value of endorectal MR imaging findings for prediction of extracapsular extension. Radiology 2004; 232: 133-9.

31 Rifkin NM, Zerhouni EA, Gatsonis CA, Quint LE, Paushter DM, et al. Comparison of magnetic resonance imaging and ultrasonography in staging early prostate cancer: results of a multiinstitutional cooperative trial. N Engl J Med 1990; 323: 621-6.

32 Hull GW, Rabbani F, Abbas F, Wheeler TM, Kattan MW, et al. Cancer control with radical prostatectomy alone in 1000 consecutive patients. J Urol 2002; 167: 528-34.

33 Heesakkers RA, Hövels AM, Jager GJ, van den Bosch HC, Witjes JA, et al. MRI with a lymph-node-specific contrast agent as an alternative to CT scan and lymph-node dissection in patients with prostate cancer: a prospective multicohort study. Lancet Oncol. 2008; 9: 850-6.

34 Harisinghani MG, Heesakkers RA, Adang EM, Jager GJ, Bar- entsz JO. Noninvasive detection of clinically occult lymphnode metastases in prostate cancer: MRI with a lymph nodespecific contrast agent compared to pelvic lymph node dissection or CT. Eur Radiol 2004; 14: 1707-12.

35 Lattouf JB, Beri A, Jeschke S, Sega W, Leeb K, et al. Laparoscopic extended pelvic lymph node dissection for prostate cancer: description of the surgical technique and initial results. Eur Urol 2007; 52: 1347-55.

36 Jemal A, Murray T, Ward E, Samuels A, Tiwari RC, et al. Cancer statistics, 2005. CA Cancer J Clin 2005; 55: 10-30. Erratum in: CA Cancer J Clin 2005; 55: 259.

37 Lentil BC, McGowan DG, Dierich H. Technetium-99 m polyphosphate bone scanning in carcinoma of the prostate. $\mathrm{Br} \mathrm{J}$ Urol 197; 46: 543-8.

38 Nozaki T, Yasuda K, Akashi T, Fuse H. Usefulness of single photon emission computed tomography imaging in the detection of lumbar vertebral metastases from prostate cancer. Int $\mathrm{J}$ Urol 2008; 15: 516-9.

39 Algra PR, Bloem JL, Tissing H, Falke TH, Arndt JW, et al. Detection of vertebral metastases: comparison between MR imaging and bone scintigraphy. Radiographics 1991; 11: 219-32. 